\title{
L'ipertensione arteriosa nel Rene Policistico Autosomico Dominante (ADPKD)
}

\section{F. Aucella}

Direttore della Struttura Complessa di Nefrologia e Dialisi, Ospedale “Casa Sollievo della Sofferenza”, Istituto di Ricovero e Cura a Carattere Scientifico, San Giovanni Rotondo

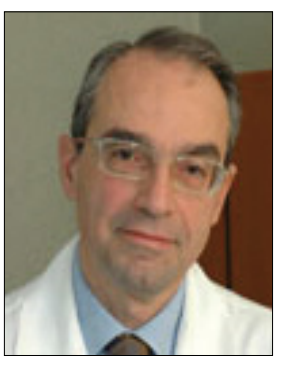

\section{Introduzione}

L'ipertensione arteriosa (IA) è il sintomo più frequente nei pazienti affetti da Rene Policistico Autosomico Dominante (ADPKD) e uno dei principali fattori che contribuisce alla progressione della malattia renale cronica nonché alla morbilità e mortalità cardiovascolare (1). $\grave{E}$ così frequente che spesso risulta la spia che fa porre la diagnosi della patologia genetica sottostante altrimenti misconosciuta, e difatti sia la perdita della funzione renale (1-3) che l'elevata frequenza di ipertrofia ventricolare sinistra $(4,5)$ correlano direttamente con i livelli pressori. Si comprende bene quindi come il raggiungimento dei target desiderati per i valori pressori sia cruciale nella gestione del paziente affetto da ADPKD per limitare la progressione verso l'uremia terminale e per la prevenzione della patologia cardiovascolare. Considerando solo i pazienti che hanno una funzione renale ancora conservata, sino a qualche anno fa un ottimale controllo pressorio era raggiunto in non più del $30 \%$ dei pazienti ipertesi con $\mathrm{ADPKD}$, ovvero un risultato simile a quello raggiunto nell'ipertensione essenziale secondo il NHANES III (6). Negli anni però la situazione è molto migliorata e la percentuale di pazienti con un buon controllo pressorio superava il $60 \%$ già nel 2000 (7) ed è probabilmente ancora maggiore oggi.

\section{Caratteristiche cliniche}

Le caratteristiche cliniche più rilevanti dell'IA nella $\mathrm{ADPKD}$ sono l'elevata frequenza, la precocità d'esordio, l'associazione con altre patologie cardiovascolari, il riscontro anche in età pediatrica e la familiarità.

\section{Prevalenza}

L'elevata frequenza di IA in corso di ADPKD è nota da tempo. Oggi è ben riconosciuto che si tratta di un riscontro tanto comune da essere diagnosticato nel $55-60 \%$ dei casi quando ancora la funzione renale è normale, a differenza di tante altre patologie nefrologiche in cui tale sintomo si manifesta solo con il declino del filtrato glomerulare. Quindi, i pazienti con ADPKD sono più spesso ipertesi rispetto a soggetti con altre patologie renali, sino a raggiungere una prevalenza che sfiora il $100 \%$ con il manifestarsi della malattia renale cronica (8).

\section{Precocità d'esordio}

L'IA non solo mostra una elevata frequenza, ma si riscontra in età più precoce nei soggetti con $\mathrm{ADPKD}$ rispetto alla popolazione generale (9). L'età media della diagnosi è di 32 anni nei maschi e di 34 anni nelle femmine, rispetto all'età media di 45-55 anni dell'IA essenziale. Ma anche se i valori di pressione arteriosa rimangono nei limiti della norma, nei giovani adulti affetti da $\mathrm{ADPKD}$ essi sono comunque più elevati che nella popolazione generale, e lo stesso andamento mostrano gli indici di 
ipertrofia ventricolare sinistra (10). Questi riscontri fanno nascere l'interessante ipotesi di un effetto benefico della terapia antipertensiva nei soggetti ancora normotesi.

\section{Associazione con altre patologie cardiovascolari}

L'IA non è l'unica patologia cardiovascolare associata alla ADPKD, accompagnandosi spesso ad altre anomalie del medesimo apparato (11). Già diversi anni fa si segnalava che ben il $18 \%$ dei soggetti e oltre il $27 \%$ di quelli di cui era disponibile anche il dato autoptico mostravano una o più patologie cardiovascolari (12), tra cui le valvulopatie della mitrale e dell'aorta erano le più frequenti. Nello studio italiano di Timio si confermava l'elevata prevalenza di patologia della mitrale, oltre il $50 \%$ dei soggetti, e della valvola aortica, circa il 18\% (13).

\section{Età pediatrica}

Inoltre, diversi studi hanno dimostrato che l'IA non è appannaggio unicamente dell'età adulta, ma mostra una elevata prevalenza, dal 20 al 30\%, anche nell'adolescenza. In una casistica di 154 adolescenti di 84 famiglie con ADPKD Sedman (14) ha segnalato che il $22 \%$ mostrava IA al momento della diagnosi, rispetto al $5 \%$ dei coetanei non affetti. A conferma, i valori di pressione arteriosa riscontrati durante monitoraggio ambulatoriale nei ragazzi o nei giovani adulti con $\mathrm{ADPKD}$, ancora senza una diagnosi di IA, appaiono già più elevati rispetto ai controlli sani e spesso mostrano altresi uno scarso calo notturno e una esaltata risposta all'esercizio fisico (15). Se questi soggetti vengono divisi in tre gruppi, ovvero pazienti con IA conclamata, pazienti con valori pressori borderline e infine normotesi, i primi due mostrano una evidente ipertrofia ventricolare sinistra rispetto ai normotesi; ma anche questi ultimi mostrano indici di massa ventricolare maggiori nei quartili con valori pressori più elevati. Queste osservazioni nell'insieme suggeriscono che il danno d'organo si sviluppa precocemente negli affetti da ADPKD e che quindi il trattamento antipertensivo potrebbe già essere indicato negli adolescenti con ipertensione borderline (16). Infine, anche in questa età si conferma l'elevata frequenza di anomalie cardiovascolari associate, con il riscontro ecocardiografico di prolasso della mitrale nel $12 \%$ dei casi rispetto al $3 \%$ dei pari età non affetti (17).

\section{Familiarità}

La ADPKD è una malattia ereditaria e il carattere familiare è insito. Tuttavia è stato anche segnalato che la presenza di IA nei soggetti affetti e l'epoca di insorgenza risentivano anche della presenza di una medesima condizione nei familiari. Pertanto, la presenza di IA nei familiari di soggetti con $\mathrm{ADPKD}$ deve raccomandare al clinico una ricerca precoce di tale condizione (18).

\section{Patogenesi}

L'IA si sviluppa in maniera parallela alla crescita delle cisti renali il che sottolinea l'importanza delle alterazioni strutturali nella sua patogenesi. Già nel 1990 Gabow dimostrava come tra i soggetti con ADPKD gli ipertesi avessero un volume renale nettamente superiore ai non ipertesi (19). Inoltre, studi angiografici hanno chiaramente messo in evidenza come nelle fasi avanzate della malattia la vascolatura renale mostri da un lato notevoli fenomeni compressivi causati dalle formazioni cistiche, dall'altro la scomparsa di irrorazione di zone periferiche del parenchima: in queste ultime zone ischemiche si determina l'atrofia tubulare e la ialinosi glomerulare. Sia la compressione che la franca ischemia determinano una forte attivazione del sistema renina-angiotensina-aldosterone (RAAS) (8) nonché del sistema nervoso simpatico (20) (Fig. 1).

L'importanza della stimolazione del RAAS è stata poi confermata da una serie di osservazioni che riportiamo in sintesi: riscontro istologico di iperplasia delle cellule secernenti renina dell'apparato juxtaglomerulare; elevati livelli di renina nel fluido intracistico e nei tubuli, elevati livelli di renina e aldosterone sia in posizione supina che in ortostatismo rispetto ai controlli sani, precocità dell'attivazione del RAAS già prima delle manifestazioni cliniche quali IA, specialmente se stimolato da un eccessivo introito salino, efficacia degli ACEi (8).

Lo stretto legame tra introito salino e valori pressori è stato più volte confermato anche nell'ADPKD. I valori pressori e la risposta ormonale in corso rispettivamente di un alto o un basso introito di sale risultano essere sovrapponibili tra soggetti con IA essenziale o in corso di $\mathrm{ADPKD}$, suggerendo quindi che probabilmente l'attivazione del RAAS intrarenale può giocare un ruolo più importante di quello sistemico nello sviluppo dell'ipertensione in questo ultimo caso (21). D'altro canto il bilancio sodico in corso di ADPKD viene mantenuto a spese di uno spostamento a destra della curva pressione-natriuresi, ovvero a scapito di più elevati livelli pressori; il fatto poi che l'espansione plasmatica non riduce l'attività reninica conferma ancora una volta l'esaltata attività di quest'ultima (22).

Oltre al ruolo fondamentale del RAAS, è oggi ben conosciuto che nei soggetti con $\mathrm{ADPKD}$ vi è altresì una 


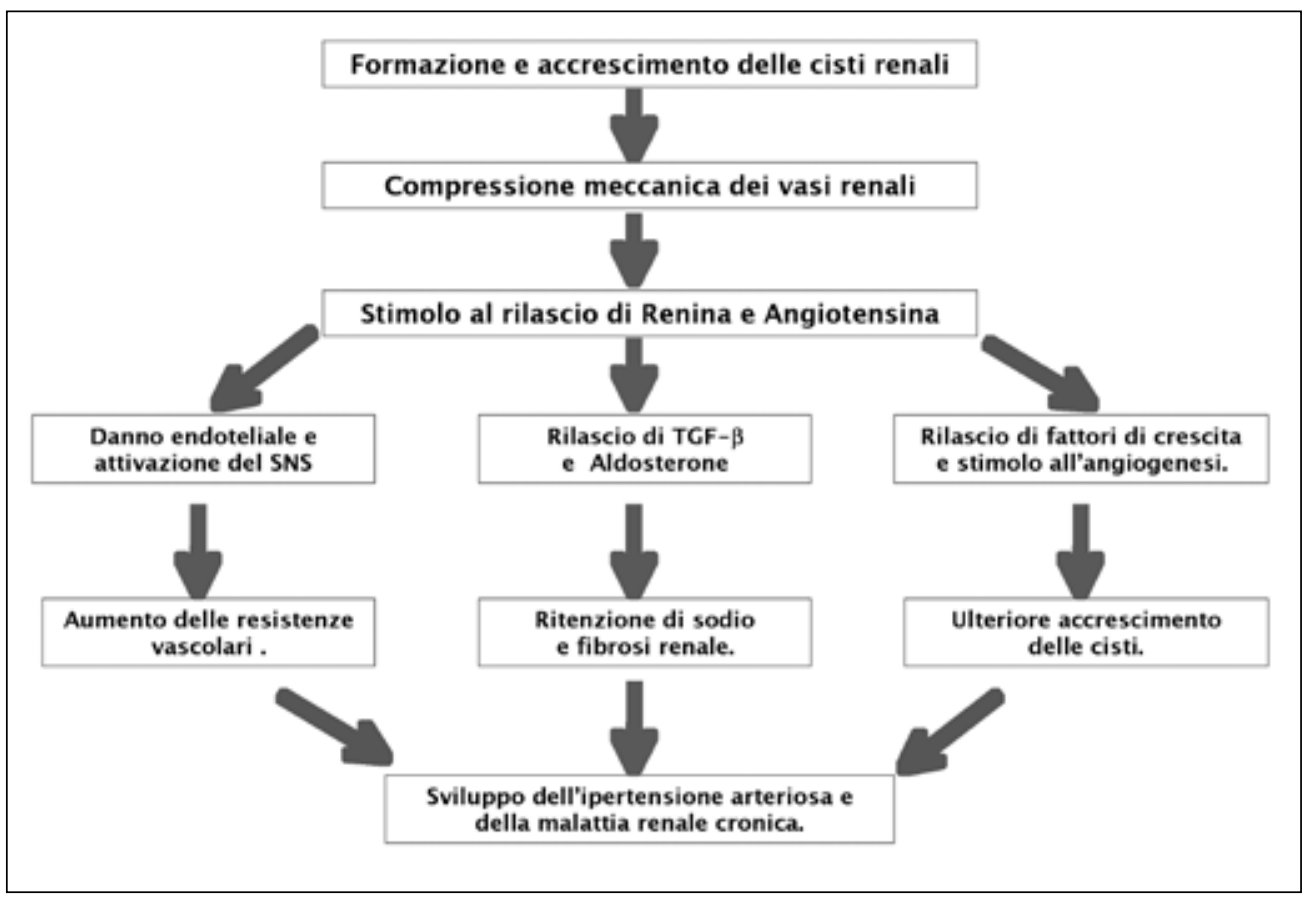

Fig. 1 - Meccanismi patogenetici dell'ipertensione arteriosa nei soggetti affetti da ADPKD (rielaborata da Ecder T, Nat Rev Nephrol 2009).

evidente e precoce attivazione del sistema nervoso simpatico, prima ancora che si instauri un deficit della funzionalità renale (20). L’iperattività simpatica aumenta il rischio cardiovascolare non solo contribuendo allincremento dei valori pressori, ma anche determinando aritmie, rimodellamento vascolare, ipertrofia ventricolare sinistra. Essa si associa inoltre a una ridotta sopravviven$z a$ in corso di uremia terminale, e pertanto rappresenta anch'essa un obiettivo della terapia cardiovascolare nel soggetto con ADPKD.

Tra i diversi altri fattori che contribuiscono allo sviluppo dell'IA, e la cui descrizione va al di là degli scopi della presente trattazione per cui si rimanda in sintesi alla Figura 1, citiamo infine il ruolo diretto delle proteine alterate dal difetto genetico sottostante. Difatti sia la policistina-1, PKD1, che la policistina-2, $\mathrm{PKD} 2$, sono regolarmente espresse nell'endotelio e nella tunica muscolare vasale, e tale espressione è alterata in corso di $\mathrm{ADPKD}(23,24)$ potendo così contribuire direttamente al determinismo delle complicanze vascolari.

\section{Terapia}

\section{Gli obiettivi della terapia}

Oggi l'obiettivo della terapia antipertensiva in questi pazienti è rappresentato da valori pressori $<120 / 80 \mathrm{mmHg}$ e dalla somministrazione precoce di inibitori del RAAS in caso di riscontro microalbuminuria o ipertrofia ventrico- lare sinistra (11) (Fig. 2). Va da sé che è indispensabile controllare tutti gli altri fattori di rischio cardiovascolare quali il tabagismo o la dislipidemia.

Se non ci sono controindicazioni il farmaco di scelta inizialmente è un inibitore dell'enzima di conversione dell'angiotensina (ACEi) o, in alternativa, un antagonista del recettore, ARB (25). Tuttavia, se il paziente è ad alto rischio è necessario uno stretto monitoraggio, specialmente in concomitanza di episodi di deplezione di volume o emorragie intracistiche.

Migliori strategie potranno essere indicate in un prossimo futuro dai risultati dello studio HALT PKD (Halt Progression of Polycystic Kidney Disease) nel quale viene valutato sia l'effetto di una terapia combinata di ACEi e $\mathrm{ARB}$ rispetto al solo $\mathrm{ACEi}$ a parità di controllo pressorio, sia se un target pressorio più stringente, (95-110/65-75 $\mathrm{mmHg}$ ) possa meglio preservare la funzione renale e/o prevenire le complicanze cardiovascolari (26).

\section{Farmaci}

Poiché nella ADPKD l'attivazione del RAAS riveste un ruolo fondamentale, e dato che già nelle malattie renali croniche, sia di origine diabetica che non, il blocco di questo sistema ha dimostrato di rallentarne l'evoluzione, molti studi hanno indagato l'efficacia di tale approccio anche nella ADPKD. I risultati tuttavia non sono stati sempre univoci e la superiorità di tale approccio è risultata, almeno sinora, parziale.

Ad esempio, nel confronto tra amlodipina, un calcio 


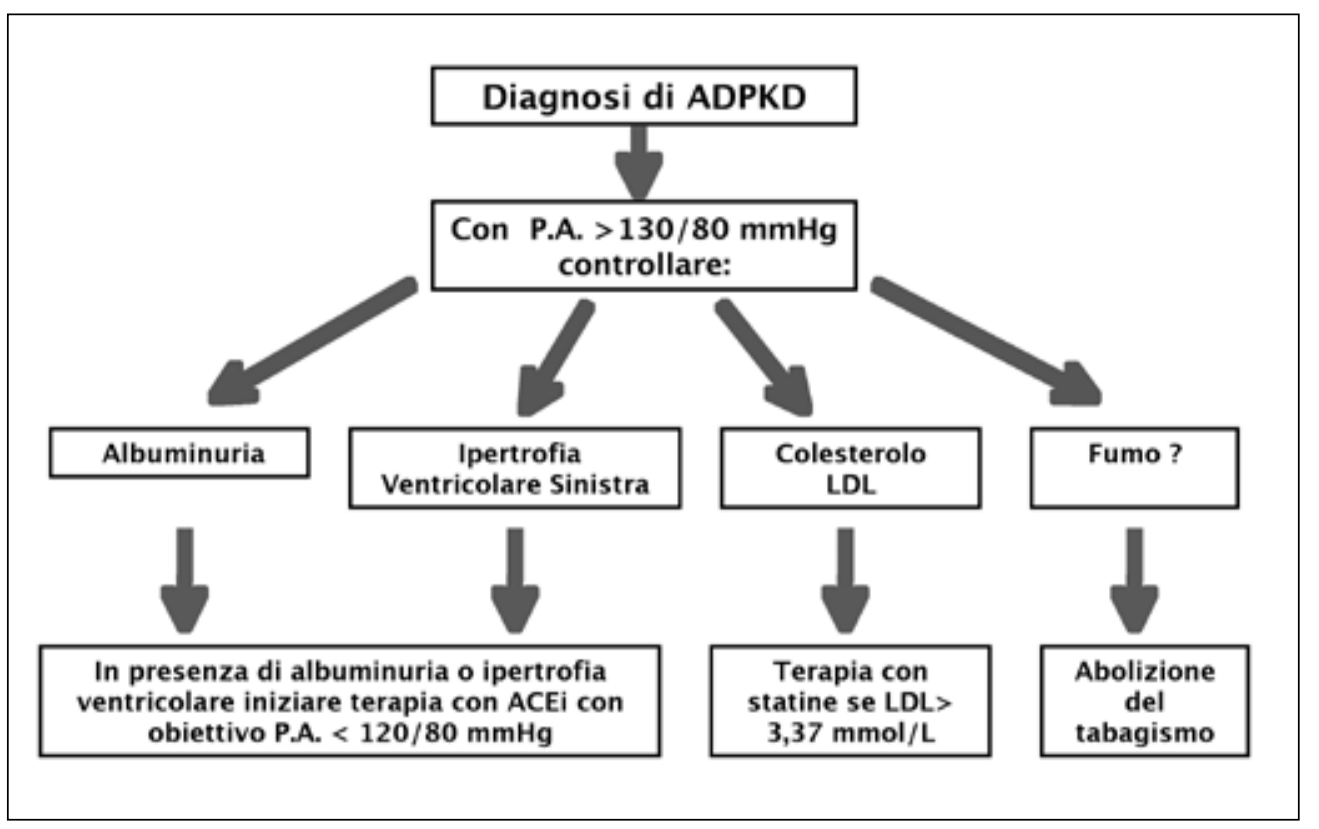

Fig. 2 - Gestione dell'ipertensione arteriosa e del rischio cardiovascolare nei soggetti affetti da ADPKD (rielaborata da Ecder T, Nat Rev Nephrol 2009).

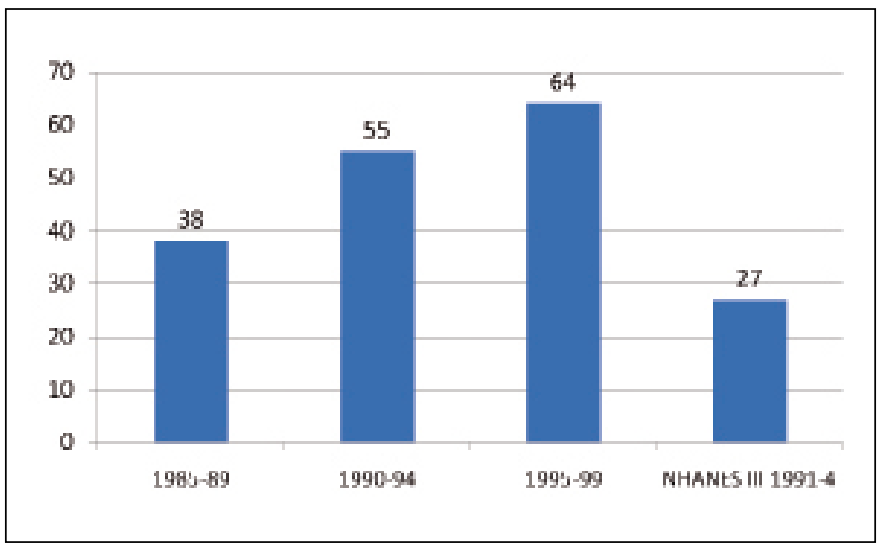

Fig. 3 - Percentuale dei pazienti affetti da ADPKD che raggiungevano un buon controllo pressorio $(<140 / 80$ mmHg): variazione negli anni e confronto con il NHANES III (rielaborata da Ecder T et al, Am J Kideny Dis 2000).

antagonista, ed enalapril, un $\mathrm{ACEi}$, alcuni Autori non riscontrarono alcuna differenza nella velocità di progressione della malattia renale cronica, benché la proteinuria si riducesse solo con il secondo farmaco (27). Ora, dato che il controllo della proteinuria si associa a una riduzione di perdita della funzionalità renale nella ADPKD, e che solo gli ACEi hanno chiaramente mostrato di controllare l'ipertrofia ventricolare sinistra (19), è possibile che l'uso degli ACEi nel lungo termine possa mostrare dei vantaggi (28). Al momento però l'aspetto fondamentale pare essere l'efficacia del controllo pressorio. Difatti un'analisi post-hoc dello studio di Zeltner et al, dove ancora una volta non vi era differenza tra $\mathrm{ACEi}$ e $\beta$-bloccanti nel preservare la funzione renale, mostrava chiaramente come i soggetti che riuscivano ad avere un rigoroso controllo pressorio, con $\mathrm{PAM}<97 \mathrm{mmHg}$, ottenevano di conseguenza una riduzione della proteinuria e un controllo dell'ipertrofia ventricolare sinistra a differenza dei soggetti che raggiungevano un "abituale" controllo dell'ipertensione arteriosa (29). Infine, anche una meta-analisi raggruppante dati di otto studi confermava che l'uso degli ACEi era sicuramente più efficace nel ridurre la proteinuria, ma che la velocità di progressione della malattia renale non era minore di quanto non riscontrato con altri farmaci (30).

Al momento, quindi, l'inibizione del RAAS non ha dimostrato un effetto benefico sulla progressione della malattia renale verso lo stadio terminale (31). Tuttavia, quando gli ACEi sono stati confrontati con i diuretici che, all'opposto, stimolano il sistema RAAS, la perdita di funzionalità renale era maggiore nei pazienti trattati con questi ultimi farmaci nonostante un controllo pressorio sovrapponibile (32); una ulteriore significativa differenza era l'aumento della proteinuria manifestatosi con l'uso dei diuretici, ma non con gli ACEi. Queste osservazioni suggeriscono che il RAAS contribuisca alla progressione della malattia renale anche indipendentemente dai valori pressori (11). La rilevanza clinica del blocco del RAAS rispetto agli altri approcci terapeutici è stata nuovamente sottolineata dagli effetti dimostrati del candesartan, un ARB che, indipendentemente dai valori pressori, risultava ridurre maggiormente sia la proteinuria che il declino del filtrato glomerulare (33). 
Nella pratica corrente, nonostante come citato non tutti gli studi dimostrino una evidente superiorità dei farmaci che bloccano il RAAS, il trattamento di questi pazienti prevede invariabilmente l'uso di un ACEi e/o di un ARB (34). Una possibile complicanza dell'inibizione del RAAS nei soggetti con ADPKD è un calo acuto e reversibile del filtrato glomerulare (35). Questa complicanza si verifica in meno del $5 \%$ dei casi e sembra essere appannaggio dei pazienti con insufficienza renale preesistente, deplezione di volume o emorragie intracistiche quali fattori di rischio addizionali. Il calo del filtrato glomerulare deriva verosimilmente dall'interferenza con l'autoregolazione vascolare in maniera del tutto simile ai casi di stenosi bilaterale dell'arteria renale: tuttavia nella ADPKD la patologia vascolare è intrarenale, causata dalla sclerosi vascolare che si accompagna alla progressione della malattia, causa o effetto dell'ipertensione arteriosa (36). Nei casi in cui si verifichi un significativo incremento dei valori di creatininemia può essere prudente passare il paziente ad altra categoria di farmaci, preferendo i $\beta$-bloccanti ai calcio-antagonisti, che possiedono un possibile effetto favorente l'accrescimento delle cisti (37). Infine, un aspetto da non sottovalutare è dato dalla necessità di trattare pazienti di sesso femminile in età fertile. In tali casi ACEi e ARB vanno banditi e la terapia deve seguire i canoni di tutte le altre pazienti gravide affette da ipertensione arteriosa.

\section{L'efficacia della terapia antipertensiva}

Quali sono quindi, in sintesi, i maggiori risultati ottenuti sinora con la terapia antipertensiva nella $\mathrm{ADPKD}$ ?

I valori pressori, se strettamente controllati, possono consentire una maggior preservazione della funzione renale, tanto da ritardarne la fase terminale, quindi l'inizio della dialisi, anche di 15 anni (7).

Per raggiungere tali obiettivi è necessaria spesso una politerapia, nell'ambito della quale, date le premesse fisiopatologiche, deve ovviamente trovare sempre posto un ACEi; questa classe di farmaci consente la riduzione del numero dei farmaci necessari (30) e la riduzione dell'ipertrofia ventricolare sinistra, fattore indipendente di rischio cardiovascolare (38).

Negli ultimi lustri si sono segnalati importanti progressi ottenuti nel controllo dell'ipertensione arteriosa nella ADPKD. Due studi epidemiologici $(7,39)$ hanno valutato il grado di controllo dei valori pressori e la sopravvivenza del rene in periodi differenti. Il primo (39) ha documentato come la percentuale di pazienti che mostravano un buon controllo dei valori pressori, inteso come PA $<140 / 80 \mathrm{mmHg}$, fosse solo il 38\% negli anni 1985-89, ma poi arrivasse al $55 \%$ e quindi al $64 \%$ nei due lustri suc- cessivi (Fig. 3). Il secondo (7) ha confrontato sia i livelli pressori che la sopravvivenza renale negli anni 19851992 e 1992-2001: nel secondo periodo i pazienti avevano valori pressori più bassi e un minor declino della funzione renale, in concomitanza con un più esteso uso degli ACEi. È ovvio che a tali risultati hanno contribuito grandemente programmi educazionali mirati sia per $\mathrm{i}$ pazienti che per i loro medici di famiglia.

\section{Conclusioni}

L'IA è una complicanza frequente e precoce nel corso della storia naturale della ADPKD e si associa a una peggiore prognosi cardiovascolare e renale. Spesso si associa l'ipertrofia ventricolare sinistra, fattore indipendente di rischio, nonché anomalie delle valvole cardiache e ulteriori manifestazioni vascolari tipiche della malattia. Un trattamento precoce ed efficace, preferibilmente con farmaci che bloccano il RAAS, se non vi sono controindicazioni, può favorevolmente influire sulla prognosi. La dimostrazione che danni vascolari precoci si instaurano già nei giovani adulti affetti, ancor prima di un evidente stato ipertensivo o di un declino della funzione renale, suggerisce che il processo aterosclerotico inizia molto presto e in maniera silente. Ne deriva la necessità di un controllo aggressivo di tutti i fattori di rischio cardiovascolari per migliorare la prognosi a lungo termine dei soggetti con ADPKD (11).

\section{Riassunto}

L'ipertensione arteriosa è il sintomo più frequente nei pazienti affetti da ADPKD e uno dei principali fattori che contribuisce alla progressione della malattia renale cronica nonché alla morbilità e mor talità cardiovascolare. Le caratteristiche cliniche più rilevanti dell'IA nella $\mathrm{ADPKD}$ sono l'elevata frequenza, la precocità d'esordio, l'associazione con altre patologie cardiovascolari, il riscontro anche in età pediatrica e la familiarità. L'obiettivo della terapia antipertensiva in questi pazienti è rappresentato da valori pressori $<120 / 80 \mathrm{mmHg}$ e dalla somministrazione precoce di inibitori del RAAS in caso di riscontro microalbuminuria o ipertrofia ventricolare sinistra. Un trattamento precoce ed efficace, preferibilmente con farmaci che bloccano il RAAS, può favorevolmente influire sulla prognosi.

Parole chiave: Rene policistico autosomico dominante, Ipertensione arteriosa, Inibitori dell'enzima di conversione, Patologia cardiovascolare 
Indirizzo degli Autori

Filippo Aucella, MD

S.C. di Nefrologia e Dialisi

Ospedale Casa Sollievo della Sofferenza IRCCS

Viale Cappuccini

71013 San Giovanni Rotondo (FG)

f.aucella@operapadrepio.it

\section{Bibliografia}

1. Gabow PA, Johnson AM, Kaehny WD, et al. Factors affecting the progression of renal disease in autosomal dominant polycystic kidney disease. Kidney Int 1992; 341: 1311-9.

2. Marcelli D, Locatelli F, Alberti D, et al. Hypertension as a factor in chronic renal insufficiency progression in polycystic kidney disease. Nephrol Dial Transplant 1995; 10(Suppl 6): S15-17.

3. Chapman AB, Schrier RW. Pathogenesis of hypertension in autosomal dominant polycystic kidney disease. Semin Nephrol 1991; 11: 653-60.

4. Gabow P, Schrier RW. Left ventricular hypertrophy in autosomal dominant polycystic kidney disease. J Am Soc Nephrol 1997; 8: 1292-7.

5. Fick GM, Johnson AM, Hammond WS, Gabow PA. Causes of death in autosomal dominant polycystic kidney disease. J Am Soc Nephrol 1995; 5: 2048-56.

6. The Joint National Committee on Prevention, Detection, Evaluation and Treatment of High Blood Pressure and the National High Blood Pressure Education Program Coordinating Committee: The Sixth Report of the Joint National Committee on Prevention, Detection, Evaluation, and Treatment of High Blood Pressure. Arch Intern Med 1997; 157: 2413-46.

7. Ecder T, Edelstein CL, Fick-Brosnahan GM, et al. Progress in Blood Pressure Control. In Autosomal Dominant Polycystic Kidney Disease American Journal of Kidney Diseases, 2000, Vol 36, No 2 (August): 266-71.

8. Ecder T, Schrier RW. Hypertension in autosomal-dominant polycystic kidney disease: early occurrence and unique aspects. J Am Soc Nephrol 2001; 12: 194200.

9. Kelleher CL, et al. Characteristics of hypertension in young adults with autosomal dominant polycystic kidney disease compared with the general U.S. population. Am J Hypertens 2004; 17: 1029-34.

10. Zeier M, Geberth S, Schmidt,KG, et al. Elevated blood pressure profile and left ventricular mass in children and young adults with autosomal dominant polycystic kidney disease. J Am Soc Nephrol 1993; 3: 1451.

11. Ecder T, Schrier RW. Cardiovascular abnormalities in autosomal-dominant polycystic kidney disease. Nat Rev Nephrol 2009; 5(4): 221-8.

12. Leier CV, et al. Cardiovascular abnormalities associated with adult polycystic kidney disease. Ann Intern Med 1984;100: 683-8.

13. Timio M, et al. The spectrum of cardiovascular abnormalities in autosomal dominant polycystic kidney disease: a 10-year follow-up in a five-generation kindred. Clin Nephrol 1992; 37: 245-51.

14. Sedman A, et al. Autosomal dominant polycystic kidney disease In childhood: a longitudinal study. Kidney Int 1987; 31: 1000-05.

15. Cadnapaphornchai MA, McFann K, Strain JD, et al. Increased left ventricular mass in children with autosomal dominant polycystic kidney disease and borderline hypertension. Kidney Int 2008; 74: 1192-6.

16. Torres VE and Harris PC. Autosomal dominant polycystic kidney disease: the last 3 years. Kidney Int 2009; 76: 149-68.

17. Ivy DD, et al. Cardiovascular abnormalities in children with autosomal dominant polycystic kidney disease. J Am Soc Nephrol 1995; 5: 2032-6.

18. Schrier RW, et al. The role of parental hypertension in the frequency and age of diagnosis of hypertension in offspring with autosomal-dominant polycystic kidney disease. Kidney Int 2003; 64: 1792-9.

19. Gabow PA, Chapman AB, Johnson AM, et al. Renal structure and hypertension in autosomal dominant polycystic kidney disease. Kidney Int 1990; 38(6): 1177-80.

20. Neumann J, Ligtenberg G, Klein IH, Blankestijn PJ. Pathogenesis and treatment of hypertension in polycystic kidney disease. Curr Opin Nephrol Hypertens 2002; 11: 517-21.

21. Doulton TW, et al. The effect of sodium and angiotensin-converting enzyme inhibition on the classic circulating renin-angiotensin system in autosomal-dominant polycystic kidney disease patients. J Hypertens 2006; 24: 939-45.

22. Torres VE, et al. Natriuretic response to volume expansion in polycystic kidney disease. Mayo Clin Proc 1989; 64: 509-15.

23. Griffin MD, et al. Vascular expression of polycystin. J Am Soc Nephrol 1997; 8: 616-26.

24. Torres VE, et al. Vascular expression of polycystin-2. J Am Soc Nephrol 2001; 12: 1-9.

25. Rizk D, Chapman AB. Cystic and inherited kidney diseases. Am J Kidney Dis 2003; 42: 1305. 
26. Chapman AB. Approaches to testing new treatments In autosomal dominant polycystic kidney disease: Insights from the CRISP and HALT-PKD studies. Clin J Am Soc Nephrol 2008; 3: 1197-204.

27. Ecder T, et al. Effect of antihypertensive therapy on renal function and urinary albumin excretion in hypertensive patients with autosomal dominant polycystic kidney disease. Am. J Kidney Dis 2000; 35: 427-32.

28. Chapman $\mathrm{AB}$, et al. Overt proteinuria and microalbuminuria In autosomal dominant polycystic kidney disease. J Am Soc Nephrol 1994; 5: 1349-54.

29. Zeltner R, et al. Renal and cardiac effects of antihypertensive treatment with ramipril vs metoprolol in autosomal dominant polycystic kidney disease. Nephrol Dial Transplant 2008; 23: 573-9.

30. Jafar TH, et al. The effect of angiotensin-converting enzyme Inhibitors on progression of advanced polycystic kidney disease. Kidney Int 2005; 67: 265-71.

31. Grantham JJ, Chapman AB, Torres VE. Volume progression in autosomal dominant polycystic kidney disease: The major factor determining clinical outcomes. Clin J Am Soc Nephrol 2006: 1: 148-57.

32. Ecder T, et al. Diuretics versus angiotensin-converting enzyme inhibitors In autosomal dominant polycystic kidney disease. Am J Nephrol 2001; 21: 98-103.

33. Nutahara K, et al. Calcium channel blocker versus angiotensin II receptor blocker in autosomal dominant polycystic kidney disease. Nephron Clin Pract 2005; 99: c18-c23.

34. Chobanian AV, Bakris GL, Black HR, et al. The Seventh Report of the Joint National Committee on Prevention, Detection, Evaluation, and Treatment of High Blood Pressure: The JNC 7 report. JAMA 2003; 289: 2560-72.

35. Chapman, AB, Gabow, PA, Schrier, RW. Reversible renal failure associated angiotensin-converting enzyme inhibitors in polycystic kidney disease. Ann Intern Med 1991; 115: 769.

36. Zeier, M, Fehrenbach, P, Geberth, S, et al. Renal histology in polycystic kidney disease with incipient and advanced renal failure. Kidney Int 1992; 42: 1259.

37. Sarnak MJ, Greene T, Wang X, et al. The effect of a lower target blood pressure on the progression of kidney disease: long-term follow-up of the modification of diet in renal disease study. Ann Intern Med 2005; 142: 342.

38. Ecder T, Edelstein CL, Chapman AB, et al. Reversal of left ventricular hypertrophy with angiotensin converting enzyme inhibition in hypertensive patients with autosomal dominant polycystic kidney disease. Nephrol Dial Transplant 1999;14(5): 1113-6.

39. Schrier RW, et al. Epidemiological study of kidney survival in autosomal dominant polycystic kidney disease. Kidney Int 2003; 63: 678-85. 